\title{
Rhupus : Étude de Deux Observations à Conakry, Guinée
}

\author{
Condé Kaba, \\ Service de Rhumatologie, CHU Ignace Deen, \\ Université de Conakry, Guinée \\ Garba Mahaman Salissou, \\ Service de Rhumatologie Centre Hospitalier Régional de Maradi, \\ Université de Niamey, Niger \\ Niasse Moustapha, \\ Diallo Rama, \\ Service de Rhumatologie CHU Aristide Le Dantec, \\ Université de Dakar, Sénégal
}

Doi: 10.19044/esj.2019.v15n12p181 URL:http://dx.doi.org/10.19044/esj.2019.v15n12p181

\section{Résumé}

Le rhupus est une association de polyarthrite rhumatoïde et de lupus érythémateux systémique. Il s'agit d'une entité clinique rare qui reste controversée. Nous en rapportons deux nouvelles observations de patients chez un homme de 48 ans et une femme de 26 ans qui illustrent, en confrontation avec les données de la littérature, la réalité de son existence. Le diagnostic de PR (Polyarthrite rhumatoïde) et de LES (lupus érythémateux systémique) était porté sur des arguments cliniques et paracliniques en accord avec les critères ACR (American College of Rheumatology) / EULAR (European League Against Rheumatism) 2010 pour la PR et ACR 1997 pour le LES. L'association de la polyarthrite rhumatoïde et du lupus est rare mais réelle.

Mots-clés : Polyarthrite rhumatoïde, lupus, rhupus 


\title{
Rhupus: Two Observations Study in Conakry, Guinea
}

\author{
Condé Kaba, \\ Service de Rhumatologie, CHU Ignace Deen, \\ Université de Conakry, Guinée \\ Garba Mahaman Salissou, \\ Service de Rhumatologie Centre Hospitalier Régional de Maradi, \\ Université de Niamey, Niger \\ Niasse Moustapha, \\ Diallo Rama, \\ Service de Rhumatologie CHU Aristide Le Dantec, \\ Université de Dakar, Sénégal
}

\begin{abstract}
Rhupus is a combination of RA and SLE clinical manifestations. It is a rare clinical entity which still remains controversial. We reported two (2) clinical cases which illustrate its existence by taking into account the review of medical literature. The diagnosis of both diseases was reached according to 2010 EULAR (European League Against Rheumatism)/ACR (American College of Rheumatology) classification criteria for RA and the 1997 ACR classification criteria for SLE. Although it is rare, the association of the two diseases is a real entity.
\end{abstract}

Keywords: Rheumatoid arthritis, lupus, rhupus

\section{Introduction}

La polyarthrite rhumatoïde (PR) et le lupus érythémateux systémique (LES) sont des maladies inflammatoires auto-immunes chroniques présentant différentes caractéristiques cliniques et sérologiques, affectant spécifiquement les articulations ou au contraire différents organes ou systèmes (McInnes et al., 2011; Rees et al., 2016). Ces maladies se distinguent habituellement selon des critères bien établis (Rees et al., 2016).

L'association PR et LES a été décrite pour la première fois par Toone en 1960 (Toone et al., 1960) et en 1971, Shur rapporte le premier cas sous le terme de rhupus (Shur et al., 1971). En général plus de 150 observations ont été rapportées dans le monde avec un taux de prévalence estimé à environ 
0,09\% (Sarkar et al., 2012). En ce qui concerne cet article, nous rapportons deux présentations différentes de rhupus.

\section{Observations \\ Observation 1.}

Nous rapportons le cas d'un patient de 48 ans connu pour un lupus érythémateux systémique depuis 2008 sur base d'atteintes cutanées et immunologiques, répondant aux critères de l'American College of Rheumatology (ACR) 1997 et traité par Plaquenil. En octobre 2014, il est hospitalisé dans le service de rhumatologie pour une polyarthrite chronique périphérique bilatérale et symétrique, non déformante, non ankylosante intéressant les épaules, les poignets, les métacarpo-phalangiennes, les interphalangiennes proximales, les genoux et les chevilles, évoluant depuis 8 ans par poussées entrecoupées de rémissions, associée à un érythème du visage et du tronc caractérisé par une photosensibilité. Il n'était retrouvé ni d'amaigrissement, ni d'alopécie, ni de sècheresse buccale et oculaire. À l'examen physique, le patient est en bon état général, il ne présente pas de fièvre. Gonflement des articulations métacarpo-phalangiennes 1 et 3 bilatérales, des interphalangiennes proximales 2 et 3 droites du poignet bilatéralement. Un érythème du visage en vespertilio et de la poitrine.

La biologie retrouvait une numération formule sanguine normale, un syndrome inflammatoire biologique non spécifique avec une vitesse de sédimentation (VS) accélérée à $52 \mathrm{~mm}$ à la $1^{\text {ère }}$ heure et une protéine $\mathrm{C}$ réactive $(\mathrm{CRP})$ positive à $11.3 \mathrm{mg} / \mathrm{l}$. La protéinurie des 24 heures était de 0.63 $\mathrm{g} /$ jour. Les fonctions rénale et hépatique étaient normales.

Sur le plan immunologique, les anticorps anti-nucléaires (AAN) étaient positifs à 1/320 de fluorescence mouchetée, de même que les anticorps anti-dsDNA natifs (209 U/ml), les anticorps anti-peptides cycliques citrullinés (300 U/ml), les antis coagulants lupiques, et les antiphospholipides (337 UGPL, recontrôlée douze semaines plus tard). Il y’avait une hypocomplémentémie CH50 à $29.52 \mathrm{U} / \mathrm{ml}$ (normale 41-94). Les facteurs rhumatoïdes (FR) et les anticorps anti-Sm étaient négatifs.

Le CT cone beam des mains/poignets montrait la présence d'une carpite stade II à droite et stade III à gauche et ausssss niveau des pieds, aussi bien que des érosions de l'os cuboïde droit. Le diagnostic de rhupus a été ainsi retenu devant ce tableau de polyarthrite chronique érosive, la positivité des anti-CCP et des anti-dsDNA et l'hypocomplémentémie. En outre, nous retrouvions un score de 10/10 pour les critères de l'ACR/EULAR pour la PR et 4 critères de l'ACR 1997 pour le LES. Après l'échec des DMARDs ( méthotrexate, hydroxychloroquine), du medrol $4 \mathrm{mg} /$ jour et de l'abatacept, le patient a été traité par Rituximab (Mabthera) avec une bonne évolution clinique dès le deuxième mois. Après un recul de 6 mois, l'HAQ (Health 
Assessment Questionnaire) était de 15/60, le DAS-28 à 3,1 et le SLEDAI (Systemic Lupus Erythematosus Disease Activity Index) à 6.

\section{Observation 2.}

Il s'agissait d'une patiente de 26 ans reçue en consultation de rhumatologie pour une polyarthrite chronique polysynoviale évoluant depuis 5 ans avec des poussées entrecoupées de rémissions, déformante et ankylosante touchant de façon symétrique et bilatérale les coudes, les poignets, les métacarpo-phalangiennes, les chevilles et les métatarsophalangiennes. La patiente avait des antécédents personnels d'éruption cutanée, de photosensibilité et d'ulcérations buccales. L'examen physique lors de la consultation montrait au niveau articulaire des synovites bilatérales et symétriques des mains, des coudes, des chevilles et des pieds avec un flessum irréductible des coudes droit> gauche et une boiterie liée à une impotence fonctionnelle des pieds. Par ailleurs le reste de l'examen physique est normal à l'exception d'un érythème au décolleté (face antérieure du thorax).

La biologie montrait une numération formule sanguine normale, un syndrome inflammatoire avec une vitesse de sédimentation normale à 16 $\mathrm{mm} /$ heure, mais une protéine C-réactive positive à $22.6 \mathrm{mg} / \mathrm{l}$. Les facteurs rhumatoïdes (FR) étaient négatifs, alors que les anticorps anti-CCP (peptides cycliques citrullinés) étaient positifs à $62 \mathrm{U} / \mathrm{ml}$. La recherche des anticorps antinucléaires (ANA) était positive à 1/80 en immunofluorescence sur cellules Hep-2, avec une positivité des anticorps anti-ADN (acide désoxyribonucléique.) double brins positif à $300 \mathrm{U} / \mathrm{ml}$. Cependant, l'identification des anticorps anti-ENA (anti-RNP, anti-Sm, anti-SSA, antiSSB, Jo1, Scl 70, centromère) étaient négatifs. On notera l'absence de consommation ou de déficit en complément $\mathrm{C} 3$ et $\mathrm{C} 4$ ou de cryoglobulinémie. Bien que n'ayant pas d'antécédent de maladie thromboembolique ou d'histoire faisant suspecter un syndrome des antis phospholipides, une mise au point de la coagulation s'est révélée normale sans trouble plaquettaire ; on notera l'absence d'antis coagulants lupiques, mais des anticorps anti phospholipides étaient positifs à 21.3 UGPL.

La radiographie des mains et des pieds était normale. Par contre, le CT cone beam des mains retrouvait la présence d'une érosion marginale au niveau de la base du deuxième métacarpien et de la partie inférieure du trapézoïde.

Le diagnostic de rhupus a été posé devant ce tableau, d'autant plus que le score ACR/EULAR pour la PR était de 7/10 et la présence de 5 critères de l'ACR 1997 pour le lupus. Le traitement était basé sur le méthotrexate, l'hydroxychloroquine et le medrol pendant 7 mois avec une bonne amélioration, l'HAQ 10/60, le DAS-28 3.6 et le SLEDA à 5. 


\section{Discussion}

Le rhupus est défini par l'association d'une polyarthrite érosive caractéristique de la PR et de manifestations cliniques évocatrices du lupus avec la présence d'anticorps spécifiques à ces deux entités (anti-CCP pour la PR et anti-dsDNA natifs et/ou anti-Sm pour le LES) (Sarkar et al., 2012). Il s'agit d'une entité dont l'existence même reste controversée. Nous en rapportons deux cas chez un homme et une femme. Le rhupus concerne surtout la femme jeune (Amezcua-Guerra, 2006).

Le LES et la PR ont été longtemps considérés comme deux connectivites mutuellement exclusives. Cette affirmation est soutenue par les profils cliniques, génétiques et pathogéniques différents des deux maladies. En effet, les manifestations auto-inflammatoires au cours de la PR font intervenir la voie Th1, tandis que le LES fait intervenir la voie Th2 (AmezcuaGuerra, 2006). Cette association a une incidence très faible $(0,01 \%-0,2 \%)$ chez les patients atteints d'arthrite (Hayakawa et al., 2007) et le taux de prévalence est estimée à 0,09 (Shur et al., 1971). Certains auteurs considèrent que le rhupus est un état de chevauchement entre PR et LES puisque les antiCCP qui sont hautement spécifiques de la PR et les anti-dsDNA/Sm qui sont hautement spécifiques du LES existent tous les deux dans le rhupus (Shur et al., 1971 ; Fernandez et al., 2007). Cependant, il existe une auto-immunité partagée dans la pathogenèse de la PR et du LES. Le gène TAP2*0201 et les variantes de gènes TNF-308A dans la même région chromosomique augmentent la susceptibilité aux maladies auto-immunes telles que la PR, le LES et le syndrome de Sjögren (Alfadhli et al., 2014). Il est démontré que les allèles HLA-DR1 et HLA-DR2 sont augmentés de façon significative chez les patients présentant un rhupus (Fernandez et al., 2007 ; Anaya et al., 2006).

Chez les patients lupiques, le caractère homozygote pour l'épitope partagé multiplie par 8 le risque d'érosion osseuse (Orozco et al., 2006). Des anticorps anti-CCP ont été détectés dans $2 \%$ des LES, et ce pourcentage monte jusqu'à 20 à $80 \%$ si le lupus est érosif ou s'il y'a présence d'arthrites (Chan et al., 2008 ; Mediwake et al., 2001). La présence d'anticorps anti-CCP chez ces patients lupiques était également associée à un risque d'érosion osseuse significativement augmenté (Martínez et al., 2007 ; Budhram et al., 2014).

Le diagnostic de PR précède les manifestations du LES et très rarement les signes de PR et du LES sont présents de façon simultanée. Cependant ce sont les signes cliniques de la PR qui dominent (Sarkar et al., 2012).

\section{Conclusion}

Le rhupus est une entité clinique rare caractérisée par l'association du lupus et de la polyarthrite rhumatoïde. La question reste encore posée si le 
rhupus est un chevauchement, un sous-groupe de lupus à expression articulaire intense ou une entité différente?

\section{Déclaration de liens d'intérêts}

Les auteurs déclarent ne pas avoir de liens d'intérêts.

\section{References:}

1. AlFadhli, S. \& Nizam, R. (2014). Rhupus: a crosswalk between lupus and rheumatoid arthritis. OA Arthritis; 2 (1): 3.

2. Amezcua-Guerra, LM., Springall, R., Marquez-Velasco, R. et al. (2006). Presence of antibodies against cyclic citrullinated peptides in patients with "rhupus": a cross-sectional study. Arthritis Res Ther ; 8 : R144.

3. Anaya, JM., Gomez, L., \& Castiblanco, J. ( 2006). Is there a common genetic basis for autoimmune diseases? Clinical and Developmental Immunology; 13 (2 -4): 185-195.

4. Budhraml, A., Chu, R., Rusta-Sallehy, S. et al. (2014). Anti-cyclic citrullinated peptide antibody as a marker of erosive arthritis in patients with systemic lupus erythematosus: a systematic review and metaanalysis. Lupus; 23; 1156-1163.

5. Chan, MT., Owen, P., Dunphy, J. et al. (2008). Associations of erosive arthritis with anti-cyclic citrullinated peptide antibodies and MHC Class II alleles in systemic lupus erythematosus. J Rheumatol; 35: 7783.

6. Fernandez, A., Quintana, G., Matteson, EL., et al. (2004). Lupus arthropathy: historical evolution from deforming arthritis to rhupus. Clin Rheumatol; 23: 523-526.

7. Hayakawa, S., Komine-Aizawa, S., Osaka, S. et al. (2007). Rembrandt's Maria Bockenolle has a butterfly rash and digital deformities: overlapping syndrome of rheumatoid arthritis and systemic lupus erythematosus. Medical hypotheses; 68(4) :906-9.

8. Martinez, JB., Valero, JS., Bantista, AJ. et al. (2007). Erosive arthropathy: clinical variance in lupus erythematosus and association with anti-CCP case series and review of the literature. Clin Exp Rheumatol; 25: 47-53.

9. McInnes, IB. \& Schett, G. (2011). The pathogenesis of rheumatoid arthritis. New England Journal of Medicine; 365 (23): 2205-2219.

10. Mediwake, R., Isenberg, DA., Schellekens, GA. et al. (2001). Use of anti-citrullinated peptide and antiRA33 antibodies in distinguishing erosive arthritis in patients with systemic lupus erythematosus and rheumatoid arthritis. Annals of Rheumatic Disease; 60: 67-8. 
11. Orozco, G., Eyre, S., Hinks, A. et al. (2011). Study of the common genetic background for rheumatoid arthritis and systemic lupus erythematosus. Annals of the Rheumatic Diseases; 70 (3): 463-468.

12. Rees, F., Doherty, M., Grainge, M. et al. (2016). The incidence and prevalence of systemic lupus erythematosus in the UK, 1999-2012. Annals of the Rheumatic Diseases; 75 (1):136-141.

13. Sarkar, S. \& Saha, K. (2012). Bilateral acute lupus pneumonitis in a case of rhupus syndrome. Lung India; 29 (3): 280-282.

14. Schur, PH. (1971). Systemic lupus erythematosus in Cecil-Loeb Texbook of Medicine. Philadelphia, PA: 1971

15. Toone, E., Irby, R., \& Pierce, EL. (1960). The cell LE in rheumatoid arthritis. American Journal of Medicine Sciences; 240:599-608. 